\title{
HOLOMORPHY ON SPACES OF DISTRIBUTION
}

\section{Philip J. Boland and Seân Dineen}

If $E$ is a locally convex space and $U \subset E$ is open, then $H(U)$ is the space of holomorphic functions on $U$ (i.e., $H(U)=$ $\left\{f: U \rightarrow C, f G\right.$-analytic and continuous). $\tau_{0}$ is the topology of uniform convergence on compact subsets of $U . \tau_{\omega}$ is the Nachbin ported topology defined by all semi-norms on $H(U)$ ported by compact subsets of $U$. (A semi-norm $p$ on $H(U)$ is ported by $K$ if whenever $V$ is open and $K \subset V \subset U$, there exists $C_{V}$ such that $p(f) \leq C_{V}|f|_{V}$ for all $f \in H(U)$.) $\tau_{\delta}$ is the topology defined by all semi-norms $p$ on $H(U)$ with the following property: if $\left(U_{n}\right)$ is a countable increasing open cover of $U$, there exist $C>0$ and $U_{N}$ such that $p(f) \leqq C|f|_{v_{N}}$ for all $f \epsilon$ $H(U) . \quad H_{H Y}(U)$ is the space of hypoanalytic functions on $U-$ that is $H_{B Y}(U)=\{f: f$ is $G$-analytic and the restriction of $f$ to any compact set $K \subset U$ is continuous\}.

If $\Omega$ is open in $R^{n}$, then $\mathscr{D}(\Omega)$ and $\mathscr{D}^{\prime}(\Omega)$ are respectively the Schwartz space of test functions and the Schwartz space of distributions on $\Omega$. We prove that $H(\mathscr{D}(\Omega)) \neq H_{H Y}(\mathscr{D}(\Omega)$ ) and that $\tau_{0}=\tau_{\omega}=\tau_{\delta}$ on $H(\mathscr{D}(\Omega))$ while $H_{H Y}\left(\mathscr{D}^{\prime}(\Omega)\right)=H\left(\mathscr{D}^{\prime}(\Omega)\right)$ but $\tau_{0} \neq \tau_{\omega} \neq \tau_{\delta}$ on $H\left(\mathscr{D}^{\prime}(\Omega)\right)$.

1. Holomorphic and hypoanalytic functions on countable direct sums. In this section, we will prove two lemmas which are useful in the construction of holomorphic and hypoanalytic functions on countable direct sums. If $E_{n} \neq 0$ is a locally convex space for each $n$, then $E=\sum_{n=0}^{\infty} E_{n}$ is the countable direct sum of the $E_{n}$ with the finest locally convex topology such that $E_{n} \rightarrow E$ is continuous for all $n$. $\Pi_{n=0}^{\infty} E_{n}$ is the product of the $E_{n}$ with the product topology.

LEMma 1. Let $E=\sum_{n=0}^{\infty} E_{n}=E_{0} \oplus E_{1} \oplus E_{2} \oplus \cdots$ where each $E_{n} \neq 0$. For each $n>0$, let $\psi_{n} \in E_{n}^{\prime}, \psi_{n} \neq 0$. Let $\left(\phi_{n}\right)_{n=1}^{\infty} \subseteq E_{0}^{\prime}$. Then $p=\sum_{n=1}^{\infty} \phi_{n} \psi_{n} \in P_{H Y}\left({ }^{2} E\right)$ (i.e., is a hypocontinuous polynomial of degree 2 on $E)$, and $p \in P\left({ }^{2} E\right)$ if and only if there exists an absolutely convex neighborhood $V_{0}$ of zero in $E_{0}$ such that $\left|\phi_{n}\right|_{V_{0}}<+\infty$ for each $n$, (i.e., $\left.\left(\phi_{n}\right)_{n=1}^{\infty} \subseteq E_{0}^{\prime}\left(V_{0}\right)\right)$.

Proof. Since each compact subset of $E$ is contained in $E_{0} \oplus \cdots$ $\oplus E_{m}$ for some $m$, it follows that $p$ is a hypoanalytic polynomial of degree 2. Now if $p$ is continuous $\left(p \in P\left({ }^{2} E\right)\right)$ there exists an absolutely convex neighborhood of zero $V=V_{0} \oplus V_{1} \oplus V_{2} \oplus \cdots$ such that $|p|_{V} \leqq$ 1. Now if $n \geqq 1$ is given and $y_{n} \in V_{n}$ is such that $\psi_{n}\left(y_{n}\right) \neq 0$, then $\left|\phi_{n}\right|_{v_{0}} \leqq 1 /\left|\psi_{n}\left(y_{n}\right)\right|$. 
Conversely suppose $V_{0}$ is an absolutely convex neighborhood of zero in $E_{0}$ such that for each $n,\left|\phi_{n}\right|_{V_{0}} \leqq C_{n}$. Now choose $V_{n}$ an absolutely convex neighborhood of zero in $E_{n}$ such that $\left|\psi_{n}\right|_{V_{n}} \leqq$ $1 / C_{n} 2^{n}$. Then $p$ is bounded on the neighborhood $V=V_{0} \oplus V_{1} \oplus V_{2} \oplus \cdots$ of zero in $E$ and hence $p$ is continuous at zero. Similarly, one may show that $p$ is bounded on a neighborhood of an arbitrary point, and hence $p$ is continuous on $E$.

Corollary 2. Let $E=E_{0} \oplus E_{1} \oplus E_{2} \oplus \cdots=\sum_{n=0}^{\infty} E_{n}$ where $E_{0}$ is a metrizable nonnormed space. Then $P\left({ }^{2} E\right) \neq P_{H Y}\left({ }^{2} E\right)$, and hence $H(E) \neq H_{H Y}(E)$.

Proof. Since $E_{0}$ is metrizable and nonnormed, there exists a neighborhood basis $\left(W_{n}\right)$ of zero in $E_{0}$ and a sequence $\left(\phi_{n}\right)$ of continuous linear forms on $E_{0}$ such that $\left|\phi_{n}\right|_{W_{n}}<+\infty$, but $\left|\phi_{n}\right|_{W_{n-1}}=$ $+\infty$ for each $n$. Hence, constructing $\left(\phi_{n}\right)$ as in Lemma 1 , we see $p=\sum_{n=1}^{\infty} \phi_{n} \psi_{n} \in P_{H Y}\left({ }^{2} E\right) \backslash P\left({ }^{2} E\right)$.

LEMmA 3. Let $E=E_{1} \oplus E_{2} \oplus E_{3} \oplus \cdots=\sum_{n=1}^{\infty} E_{n}$. Suppose $f_{n} \epsilon$ $H\left(E_{n}\right), f_{n}(0)=0$ for each $n$, and let $g_{n}$ be a continuous polynomial on $E_{1} \oplus \cdots \oplus E_{n}$ (i.e., $g_{n} \in P\left(E_{1} \oplus \cdots \oplus E_{n}\right)$ ) for each $n$. Then if there exists a neighborhood $U$ of zero in $E$ such that $\left|g_{n}\right|_{U}<+\infty$ for all $n$, then $F=\sum_{n=1}^{\infty} g_{n} f_{n} \in H(E)$.

Proof. By the nature of compact sets in $E$, it is clear that $F$ is always hypoanalytic (on each compact subset $K$ of $E, F$ reduces to a finite sum of analytic functions).

Now suppose $U$ is a neighborhood of zero in $E$ such that $\left|g_{n}\right|_{U}<$ $+\infty$ for each $n$. Without loss of generality we may assume $U=$ $U_{1} \oplus U_{2} \oplus U_{3} \oplus \cdots$ where $U_{n}$ is an absolutely convex neighborhood of zero in $E_{n}$ for each $n$. Now let $V_{n}$ be an absolutely convex neighborhood of zero in $E_{n}$ such that $V_{n} \subset U_{n}$ and

$$
\left|f_{n}\right|_{V_{n}} \leqq \frac{1}{2^{n}\left|g_{n}\right|_{U}}
$$

for each $n \geqq 1$. Then $V=V_{1} \oplus V_{2} \oplus \cdots$ is an absolutely convex neighborhood of zero in $E$ and

$$
|F|_{V} \leqq \sum_{n=1}^{\infty}\left|g_{n}\right|_{V}\left|f_{n}\right|_{V} \leqq \sum_{n=1}^{\infty}\left|g_{n}\right|_{U}\left|f_{n}\right|_{V_{n}} \leqq \sum_{n=1}^{\infty} \frac{1}{2^{n}}=1
$$

Hence $F$ is locally bounded and therefore continuous at zero. We now show that $F$ is also continuous at any $x \in E$.

Given $x \in E$, there exists an $m$ such that $x=x_{1}+\cdots+x_{m} \in$ $E_{1} \oplus \cdots \oplus E_{m}$. Since $g_{n}$ is a polynomial and is bounded on $U$, it 
follows that $g_{n}$ is bounded on $x+U$.

For each $n$ let $W_{n}$ be an absolutely convex neighborhood of zero in $E_{n}$ such that $W_{n} \subset U_{n}$ and

$$
\left|f_{n}\right|_{x+W_{n}}<\frac{1}{2^{n}\left|g_{n}\right|_{x+U}} .
$$

Let $W=W_{1} \oplus W_{2} \oplus \cdots \oplus W_{m} \oplus W_{m+1} \oplus \cdots$.

Then $x+W \subset x+U$, and

$$
\begin{aligned}
|F|_{x+W} & =\left|\sum_{n=1}^{\infty} g_{n} f_{n}\right|_{x+W} \\
& \leqq \sum_{n=1}^{\infty}\left|g_{n} f_{n}\right|_{x+W} \\
& \leqq \sum_{n=1}^{\infty}\left|g_{n}\right|_{x+U}\left|f_{n}\right|_{x+W_{n}}<\sum_{n=1}^{\infty} \frac{1}{2^{n}}=1 .
\end{aligned}
$$

EXAMPLE 4. Let $g_{n} \in P\left(E_{1} \oplus \cdots \oplus E_{n}\right)$ for each $n$, and $f_{n} \in H\left(E_{n}\right)$ such that $f_{n}(0)=0$ for each $n$. Then if $E_{n}$ is normed for each $n, F=$ $\sum_{n=1}^{\infty} g_{n} f_{n} \in H(E)$.

EXAMPLE 5. Let $\phi_{n} \in E_{n}^{\prime}$, and $f_{n} \in H\left(E_{n}\right)$ for each $n$ where $f_{n}(0)=$ 0. Then $F=\sum_{n=1}^{\infty} \phi_{1} \cdots \phi_{n} f_{n} \in H(E)$.

2. Holomorphic and hypoanalytic functions on $\mathscr{D}$ and $\mathscr{D}^{\prime}$. If $\Omega$ is an open subset of $R^{n}, \mathscr{D}(\Omega)$ and $\mathscr{D}^{\prime}(\Omega)$ are respectively the Schwartz space of test functions and the Schwartz space of distributions on $\Omega$. The topologies on these spaces are normally described in terms of inductive and projective limits. Valdivia [7] has shown that for any $n$ and any open $\Omega \subset R^{n}, \mathscr{D}(\Omega) \cong \sum_{(N)} s$ and $\mathscr{D}^{\prime}(\Omega) \cong$ $\Pi_{(N)} s^{\prime}$ (where $s$ is the Fréchet nuclear space of rapidly decreasing sequences and $s^{\prime}$ is its dual). As we will see, this simplifies considerably the study of holomorphic functions on these spaces. Because of this characterization of Valdivia, we will use the notation $\mathscr{D}$ and $\mathscr{D}^{\prime}$ in place of respectively $\mathscr{D}(\Omega)$ and $\mathscr{D}^{\prime}(\Omega)$. Hence we will write $\mathscr{D} \cong \sum_{(N)} s$ and $\mathscr{D}^{\prime} \cong \Pi_{(N)} s$.

In this section, we will show that $H(U) \neq H_{H Y}(U)$ for $U$ open in $\mathscr{D}$ but that $H(U)=H_{H Y}(U)$ for $U$ open in $\mathscr{D}^{\prime}$.

Since $\mathscr{D} \cong \sum_{(N)} s$, where $s$ is the (nonnormed) Fréchet nuclear space of rapidly decreasing sequences, we see that by Corollary 2, $P\left({ }^{2} \mathscr{D}\right) \neq P_{H Y}\left({ }^{2} \mathscr{D}\right)$ and hence $H(U) \neq H_{H Y}(U)$ for $U$ open in $\mathscr{D}$.

Proposition 6. Let $U$ be open in $\mathscr{D}^{\prime}$. Then there exists a compact subset $K$ of $U$ with the property that if $f \in H_{H Y}(U)$ and $\left.f\right|_{K}=0$, then $f \equiv 0$. 
Proof. It suffices to consider the case when $U$ is an absolutely convex neighborhood of zero in $\mathscr{D}^{\prime}$. For each $n$, let $s_{n} \cong s$. Then $\mathscr{D}^{\prime} \cong \prod_{n=1}^{\infty} s_{n}^{\prime}$, and we may assume $U$ is of the form $U_{1} \times \cdots \times U_{m} \times$ $\prod_{n=m+1}^{\infty} s_{n}^{\prime}$ where $U_{n}$ is an absolutely convex neighborhood of zero in $s_{n}^{\prime}$. Since $s_{n}$ has a continuous norm, there exists a compact set $K_{n}=$ $V_{n}^{0} \subset U_{n}$ which has dense linear span in $s_{n}^{\prime}$ for each $n$. Now $K=$ $K_{1} \times K_{2} \times K_{3} \times \cdots$ is compact in $\mathscr{D}^{\prime}$ and we claim that if $f \in H_{H Y}(U)$ and $\left.f\right|_{K}=0$, then $f \equiv 0$.

If $f \in H_{H Y}(U)$ where $\left.f\right|_{K}=0$ and $f=\sum_{r=0}^{\infty} p_{r}$ is the Taylor series expansion of $f$ at 0 , it follows from the Cauchy inequalities that $\left|p_{r}\right|_{K}=0$ for each $r$. It suffices to show that $p_{r} \equiv 0$ for all $r$. Now $s_{1}^{\prime} \times \cdots \times s_{n}^{\prime}$ is a $\mathscr{D} \mathscr{F} \mathscr{N}$ space (in particular a $k$-space) and hence $\left(p_{r}\right)_{n}=\left.p_{r}\right|_{s_{1}^{\prime} \times \cdots \times s_{n}^{\prime}}$ is continuous for each $r$ and $n$. But $K_{1} \times \cdots \times K_{n}$ has dense linear span in $s_{1}^{\prime} \times \cdots \times s_{n}^{\prime}$ and thus $\left(p_{r}\right)_{n} \equiv 0$ since $\left.\left(p_{r}\right)_{n}\right|_{K_{1} \times \cdots \times K_{n}}=$ 0 . Now if $x \in \mathscr{D}^{\prime}$ and $(x)_{n}=\left(x_{1}, \cdots, x_{n}, 0, \cdots\right) \in \prod_{n=1}^{\infty} s_{n}^{\prime}$, then $(x)_{n} \rightarrow x$ and $\left\{x,(x)_{1},(x)_{2}, \cdots\right\}$ is compact. Therefore, since $p_{r}$ is continuous on compact sets, $p_{r}(x)=\lim _{n} p_{r}\left((x)_{n}\right)=\lim _{n}\left(p_{r}\right)_{n}\left((x)_{n}\right)=0$. Hence $p_{r} \equiv$ 0 for all $r$, and $f \equiv 0$.

Proposition 7. If $U$ is open in $\mathscr{D}^{\prime}$, then $H_{H Y}(U)=H(U)$.

Proof. Without loss of generality, we may assume $U$ is of the form $U_{1} \times \cdots \times U_{m} \times \prod_{n=m+1}^{\infty} s_{n}^{\prime}$ as in Proposition 6. Let $\Pi_{n}: \prod_{n=1}^{\infty} s_{n}^{\prime} \rightarrow$ $s_{1}^{\prime} \times \cdots \times s_{n}^{\prime}$ for each $n$. We claim that if $f \in H_{H Y}(U)$, then $f$ factors through some $\Pi_{n}$, i.e., there exists $n \geqq m$ and $\widetilde{f}$ a mapping from $\Pi_{n}(U)$ to $C$ making the following diagram commutative:

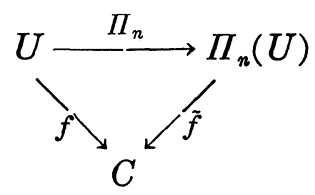

If such a factorization does exist, then clearly $\tilde{f}$ is $G$-analytic. Since $\Pi_{n}(U)$ is a $k$-space $\left(s_{1}^{\prime} \times \cdots \times s_{n}^{\prime}\right.$ is $\left.\mathscr{D} \mathscr{F} \mathscr{N}\right)$, and compact subsets of $\Pi_{n}(U)$ can be pulled back to compact subsets of $U$, it would follow that $\widetilde{f}$ and hence $f$ is continuous.

Suppose $f$ does not factor for any $n$. Then there exist two sequences $\left(x_{n}\right),\left(y_{n}\right) \subset U$ where $\Pi_{n}\left(x_{n}\right)=x_{n}, \Pi_{n}\left(y_{n}\right)=0$ and $f\left(x_{n}+y_{n}\right)-$ $f\left(x_{n}\right) \neq 0$ for each $n$.

Let $K$ be a hypoanalytically determining set for $H_{H Y}(U)$ as in Proposition 6. Now for $n>m$,

$$
g_{n}: z \longrightarrow f\left(z+y_{n}\right)-f(z) \in H_{H Y}(U)
$$

and therefore there exists $z_{n} \in K$ such that 


$$
f\left(z_{n}+y_{n}\right)-f\left(z_{n}\right) \neq 0 .
$$

Furthermore, for fixed $n>m, \lambda \rightarrow f\left(z_{n}+\lambda y_{n}\right)-f\left(z_{n}\right)$ is analytic on $\boldsymbol{C}$ and nonconstant. By Liouville's theorem there exists $\lambda_{n} \in \boldsymbol{C}$ such that $\left|f\left(z_{n}+\lambda_{n} y_{n}\right)\right|>n$. But then $f$ is unbounded on the relatively compact subset $\left(z_{n}+\lambda_{n} y_{n}\right)_{n>m}$ in $U$, contradicting the fact that $f \in$ $H_{H Y}(U)$.

CoROLLARY 8. Let $U$ be open in $\mathscr{D}^{\prime}$. Then $\tau_{0}$ bounded subsets of $H(U)$ are locally uniformly bounded (i.e., uniformly bounded in a neighborhood of each point in $U$ ).

Proof. Without loss of generality $U=U_{1} \times \cdots \times U_{m} \times \prod_{n=m+1}^{\infty} s_{n}^{\prime}$ as in Proposition 7. We proceed as in the proof of Proposition 7. If $\left(f_{\kappa}\right)_{\alpha \in A}$ is $\tau_{0}$ bounded in $H(U)$, we claim it is uniformly factorizable through some $\Pi_{n}(n>m)$. If not, we may as before find a relatively compact subset $\left(z_{r}+\lambda_{r} y_{r}\right)_{r}$ in $U$ on which $\left(f_{\alpha}\right)_{\alpha \in A}$ is not uniformly bounded. Hence $\left(f_{\alpha}\right)_{\alpha \in A}$ factors uniformly through some $\Pi_{n},(n>m)$.

But if $U \subset E$ where $E$ is a $\mathscr{D} \mathscr{F} \mathscr{N}$ space or a Fréchet space, then $\tau_{0}$ bounded subsets of $H(U)$ are locally uniformly bounded [1]. Hence $\left(\tilde{f}_{\alpha} \circ \Pi_{n}\right)_{\alpha \in A}$ is locally uniformly bounded and therefore so is $\left(f_{\alpha}\right)_{\alpha \in A}$.

3. Topologies on $H(\mathscr{D})$ and $H\left(\mathscr{D}^{\prime}\right)$. In this section, we will prove the promised results concerning the three topologies $\tau_{0}, \tau_{w}$ and $\tau_{\delta}$ on the spaces $\mathscr{D}$ and $\mathscr{D}^{\prime}$. We will use duality theory and some results already known about topologies on $H(E)$ when $E$ is a Fréchet nuclear space or a $\mathscr{D} \mathscr{F} \mathscr{N}$ space, ([3], [4], [6]).

Initially, we review some basic results in duality theory for holomorphic functions.

A locally convex space $E$ is fully nuclear if $E$ and $E^{\prime}$ are complete reflexive nuclear spaces. $\mathscr{D}, \mathscr{D}^{\prime}$ and Fréchet nuclear or $\mathscr{D} \mathscr{F} \mathscr{N}$ spaces are fully nuclear. $E$ is fully nuclear with a basis if $E$ is fully nuclear and has a Schauder (and hence absolute) basis. $\mathscr{D}$ and $\mathscr{D}^{\prime}$ are fully nuclear with a basis. If $\left(e_{n}\right)_{n=1}^{\infty}$ is a basis for the fully nuclear space $E$ and $m \in N^{(N)}$, then

$$
z=\sum_{n=1}^{\infty} z_{n} e_{n} \longrightarrow z^{m}=z_{1}^{m_{1}} \cdots z_{n}^{m_{n}}
$$

is a monomial in $z$. In [3] it is shown that if $E$ is fully nuclear with a basis, then the monomials form an absolute basis for $H(E)$ for both of the topologies $\tau_{0}$ and $\tau_{\omega}$. Furthermore, $\tau_{0}=\tau_{w}$ on $H(E)$ if and only if $\tau_{0}$ and $\tau_{\omega}$ are compatible if and only if $H_{H Y}\left(0_{E^{\prime}}\right)=$ $H\left(0_{E^{\prime}}\right)$ (the space of hypoanalytic and analytic germs at 0 in $E^{\prime}$ ). In 
particular, it follows that $\tau_{0}=\tau_{\omega}$ on $H(E)$ whenever $E$ is a Fréchet nuclear or dual of Fréchet nuclear space with a basis.

There exist Fréchet nuclear spaces with a basis $E$ where $\tau_{0}=$ $\tau_{\omega} \neq \tau_{\dot{\delta}}$ on $H(E)$. However, Dineen has shown in [6] that if $E=s$, then $\tau_{0}=\tau_{\omega}=\tau_{\delta}$ on $H(s)$. We will use this fact together with the above duality theory to show that $\tau_{0}=\tau_{\omega}=\tau_{\delta}$ on $H(\mathscr{D})$.

Proposition 9. $\tau_{0}=\tau_{\omega}=\tau_{\delta}$ on $H(\mathscr{D})$.

Proof. We have seen that $H(U)=H_{H Y}(U)$ for all $U$ open in $\mathscr{D}^{\prime}$. Hence $H\left(0_{\mathscr{O}^{\prime}}\right)=H_{H Y}\left(0_{\mathscr{D}^{\prime}}\right)$. Since $\mathscr{D}$ is a fully nuclear space with a basis, it follows by the above that $\tau_{0}=\tau_{\omega}$ on $H(\mathscr{D})$. We will now show that $\tau_{0}=\tau_{\delta}$ on $H(\mathscr{D})$. Initially however, we will introduce some further notation concerning $\mathscr{D} \cong \sum_{n=1}^{\infty} s_{n}$.

For each $n$, let $\left(e_{i n}\right)_{i}$ be an absolute basis for $s_{n}$. Then if $z=\left(z_{n}\right) \in$ $\mathscr{D}$, each $z_{n}$ is of the form $z_{n}=\left(z_{i n}\right)_{i}$. If $m_{i} \in N^{(N)}$ then $z_{i}^{m_{i}}=\boldsymbol{z}_{i 1}^{m_{11}{ }_{12} \boldsymbol{z}_{i 2}} \cdots$. Now let $m=\left(m_{1}, m_{2}, \cdots\right) \in \sum_{(N) N}(N)$. Then $z \in \mathscr{D} \rightarrow \boldsymbol{z}^{m}=\boldsymbol{z}_{1}^{m_{1}} \boldsymbol{z}_{2}^{m_{2}} \cdots$ is a monomial, and the set of monomials form a basis for $H(\mathscr{D}), \tau_{0}=$ $H(\mathscr{D}), \tau_{\omega}$. Note that a monomial on $\mathscr{D}$ is simply a finite product of monomials on $s$.

Now let $p$ be a $\tau_{\delta}$ continuous semi-norm on $H(\mathscr{D})$. We wish to show that $p$ is a $\tau_{0}$ continuous semi-norm. It suffices to show that $p$ is the extension of a $\tau_{\delta}$ continuous semi-norm on $H\left(s_{1} \oplus \cdots \oplus s_{n}\right)$ for some $n$. For in this case, since $s_{1} \oplus \cdots \oplus s_{n} \cong s$, and $\tau_{0}=\tau_{\delta}$ on $H(s)([6])$, it would follow that $p$ is $\tau_{0}$ continuous.

As $p$ is $\tau_{\delta}$ continuous on $H(\mathscr{D}), p$ is of the form $p(f)=$ $\sum_{j=0}^{\infty} p_{j}\left(\hat{d}^{j} f(0) / j\right.$ !) where $p_{j}$ is a continuous semi-norm on $P\left({ }^{j} \mathscr{D}\right)$. But $\tau_{\hat{o}}=\tau_{\omega}=\tau_{0}$ on $P\left({ }^{j}(\mathscr{D})\right)$ for each $j$, and since every compact subset of $\mathscr{D}$ is contained in $s_{1} \oplus \cdots \oplus s_{n}$ for some $n$, it follows that there exists a minimal $n_{j}$ such that $\left.f\right|_{s_{1}+\cdots+s_{n_{j}}}=0 \Longrightarrow p_{j}(f)=0$ for all $f \in$ $H(\mathscr{D})$. We claim that the sequence $\left\{n_{j}\right\}_{j}$ is bounded.

If not there exist two increasing sequences of integers $\left(r_{j}\right)_{j}$ and

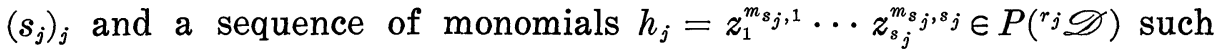
that

$$
m_{s_{j}} \neq 0 \text { and } \quad p_{r_{j}}\left(h_{j}\right) \neq 0 \text {. }
$$

Now let $k_{j}=h_{j} / p_{r_{i}}\left(h_{j}\right)$. Then $k_{j}=g_{s_{j}} f_{s_{j}}$ where $g_{s_{j}}$ is a monomial and $f_{s_{J}} \in H\left(E_{s_{j}}\right)$ such that $f_{s_{j}}(0)=0$. Now there exists a neighborhood of zero $U$ in $\sum_{n=1}^{\infty} s_{n}$ on which each monomial is bounded (each $s_{n}$ has a continuous norm). Hence if $F \equiv \sum_{j=1}^{\infty} k_{j}$, then $F \in H(\mathscr{D})$ by Lemma 3. But $p(F)=\sum_{j=1}^{\infty} p_{r_{j}}\left(k_{j}\right)=+\infty$, a contradiction. Hence $\left(n_{j}\right)_{j}$ is bounded, and there exists an $n$ such that if $f \in H(\mathscr{D}),\left.f\right|_{s_{1} \oplus \cdots \oplus s_{n}}=0$, 
then $p(f)=0$. It is clear then that $\left.p\right|_{H\left(s_{1} \oplus \cdots \oplus s_{n}\right)}$ is a $\tau_{\delta}$ continuous semi-norm on $H\left(s_{1} \oplus \cdots \oplus s_{n}\right)$ from the definition of the $\tau_{\delta}$ topology, and this completes the proof.

Proposition 10. $\tau_{0} \neq \tau_{\omega} \neq \tau_{\tilde{o}}=\tau_{0, b}=\tau_{\omega, b}$ on $H\left(\mathscr{D}^{\prime}\right)$.

Proof. We have seen that $H(\mathscr{D}) \neq H_{H Y}(\mathscr{D})$ and therefore it follows that $H\left(0_{\mathscr{A}}\right) \neq H_{H Y}\left(0_{\mathscr{O}}\right)$. Hence in particular $\tau_{0}$ and $\tau_{\omega}$ are not compatible on $H\left(\mathscr{D}^{\prime}\right)$, and therefore $\tau_{0} \neq \tau_{\omega}$.

Now $\mathscr{D}^{\prime} \cong \prod_{n=1}^{\infty} s_{n}^{\prime}$, and in particular contains a sequence $\left(y_{n}\right)_{n}\left(y_{n} \neq 0\right)$ which converges to zero very strongly (i.e., $\lambda_{n} y_{n} \rightarrow 0$ for each sequence $\left(\lambda_{n}\right)_{n}$ of complex numbers). Hence $\tau_{\omega} \neq \tau_{\omega, b}$ (bornological topology associated with $\tau_{\omega}$ ): see for example [5]. In particular $\tau_{\omega} \neq \tau_{\delta}$ since $\tau_{\hat{o}}$ is bornological [5].

Finally we show that $\tau_{0, b}=\tau_{\omega, b}=\tau_{\delta}$. Since it is easy to see from the definition of $\tau_{\delta}$ that sets of holomorphic functions which are locally uniformly bounded are $\tau_{\dot{o}}$ bounded on $H\left(\mathscr{D}^{\prime}\right)$, it suffices to show that $\tau_{0}$ bounded subsets of $H\left(\mathscr{D}^{\prime}\right)$ are locally uniformly bounded. But this is precisely Corollary 8.

REMARK 11. It is interesting to note that $\tau_{0}, \tau_{\omega}$ and $\tau_{\dot{o}}$ are three distinct nuclear topologies on $H\left(\mathscr{D}^{\prime}\right)$ ([2], [3], [6], [8]) while $\tau_{0}=\tau_{\omega}=$ $\tau_{\hat{o}}$ on $H(\mathscr{D})$ is nuclear but not sequentially complete.

Remark 12. Since $\mathscr{D}$ and $\mathscr{D}^{\prime}$ are fully nuclear with a basis, we may define open polydises in these spaces. If $U$ is an open polydisc in the fully nuclear space $E$, then $U^{M}$ (the multiplicative polar of $U$ ) is a compact subset of $E^{\prime}$. [4].

The results of Propositions 9 and 10 may be extended to show that $\tau_{0}=\tau_{\omega}=\tau_{\dot{o}}$ on $H(U)$ whenever $U$ is an open polydisc in $\mathscr{D}$ and that $\tau_{0} \neq \tau_{\omega} \neq \tau_{\dot{o}}=\tau_{0, b}=\tau_{\omega, b}$ on $H(U)$ whenever $U$ is an open subset in $\mathscr{D}^{\prime}$.

In [4] it is shown that $\tau_{0}$ is bornological on $H(U)$ if and only if the space of germs $H\left(U^{M}\right)=\lim _{V \supset U^{m}} H^{\infty}(V)$ is a regular inductive limit.

Hence if $U$ is an open polydisc in $\mathscr{D}$ (respectively $\mathscr{D}^{\prime}$ ) then $H\left(U^{M}\right)$ is regular $\left(H\left(U^{M}\right)\right.$ is not regular).

\section{REFERENBES}

1. J. A. Barroso, M. C. Matos and L. Nachbin, On bounded sets of holomorphic mappings, Proceedings on Infinite Dimensional Holomorphy, University of Kentucky 1973, Springer Verlag Lecture Notes in Mathematics, 364, 1974.

2. P. J. Boland, An example of a nuclear space in infinite dimensional holomorphy, Arkiv för matematik, 15 (1), (1977), 87-91. 
3. P. J. Boland and S. Dineen, Holomorphic Functions on Fully Nuclear Spaces, Bull. Soc. Math. France, 106 (1978), 311-336.

4. P. J. Boland and S. Dineen, Duality Theory for Spaces of Germs and Holomorphic Functions on Nuclear Spaces, Advances in Holomorphy, Ed. J. A. Barroso, North Holland Mathematics Studies, 34, 179-207.

5. S. Dineen, Holomorphic functions on locally convex topological vector spaces $I$, Locally convex topologies on $H(U)$, Ann. Inst. Fourier, 23 (1973), 1, 19-54.

6. S. Dineen, Analytic functionals on fully nuclear spaces, to appear in Studiea Math. 7. M. Valdivia, Representationes de Los Espacios $\mathscr{D}(\Omega)$ y $\mathscr{D}^{\prime}(\Omega)$, Revista Real Academia de Ciencias Exactas, Fisicas y Naturales-Madrid, 1978, 72, 385-414.

8. L. Waelbroeck, The nuclearity of $\mathcal{O}(U)$, In finite Dimensional Holomorphy and Applications, Ed. M.C. Matos, North Holland Mathematical Studies, 12 (1977), 425-634.

yeceived April 18, 1979 and in revised form December 6, 1979.

University College

Belfield, Dublin 4, Ireland 